Classification

Physics Abstracts

$05.20-36.20 \mathrm{E}-61.40 \mathrm{~K}$

\title{
Monte-Carlo simulation of multiple chain systems : screening length in two- and three-dimensional spaces
}

\author{
P. G. Khalatur, S. G. Pletneva and Yu. G. Papulov \\ Kalinin State University, Kalinin 170013, USSR
}

(Ręu le 10 mars 1982, accepté le 20 juillet 1982)

\begin{abstract}
Résumé. - Dans ce travail on étudie par la méthode de Monte-Carlo les chaînes flexibles de polymères en solution semi-diluée. On calcule la dépendance de la longueur d'écran $\xi$ en fonction de la fraction en volume $C$ du polymère à 2 et 3 dimensions. On montre que, dans les deux cas, lorsque $C$ augmente, la quantité $\xi$ décroît comme $\xi \sim C^{-b}$ où $b$ dépend de la dimensionalité $d$. On trouve que, pour $C \geqslant 0,05$ et $d=3$, la valeur de $b=0,65 \pm 0,04 \mathrm{et}$, pour $d=2, b=1,17 \pm 0,09$. Ces résultats sont qualitativement en accord avec les prédictions des lois d'échelle : $b=3 / 4(d=3)$ et $b=3 / 2(d=2)$.
\end{abstract}

\begin{abstract}
We describe the results of Monte-Carlo calculations of multichain systems with excluded volume interactions. We studied the concentration dependence of the screening length $\xi$ for threeand two-dimensional systems. It is observed that $\xi$ diminishes steadily with the increase in the concentration $C$ of chain molecules. The decrease is more pronounced for the two-dimensional system. For $C \geqslant 0.05 \xi$ is found to scale with density as $C^{-b}$, where $b=0.65 \pm 0.04$ for $d=3$ and $b=1.17 \pm 0.09$ for $d=2$. The obtained exponents are in reasonable agreement with the scaling prediction : $b=3 / 4(d=3)$ and $b=3 / 2(d=2)$.
\end{abstract}

1. Introduction. - The properties of long flexible polymer chains in dilute solution are now well understood since the discovery by de Gennes [1] of the analogy between the problems of polymer statistics and magnetic phase transitions. More recently des Cloizeaux [2] has shown that the many chain problem in a good solvent was related to the magnetic problem under an external magnetic field.

The so called blob model provides a conceptual framework to understand the features of chains in the semidilute regime of concentration where the chains overlap. The overlap or critical concentration $C^{*}$ is

$$
C^{*} \sim N / R^{d}
$$

where $R \sim a N^{v}$ is the mean end-to-end separation of a single chain ( $a$ is the effective distance between two subsequent monomers along the chain, $N$ is the polymerization index, $v$ is the excluded volume exponent. The value of $v$ is very close to the Flory-Edwards value $3 / 5$ for the three-dimensional chain $(d=3)$ [3], and is close to $3 / 4$ for the two-dimensional chain $(d=2))$.

In semidilute solutions in good solvents, each chain of $N$ monomers is viewed as made of $n$ 
blobs or subchains of size $\xi$ each containing $N_{\xi}=N / n$ monomers [4]. On scales $r<\xi$, the chain behaves like an isolated (swollen) chain. Each blob obeys the swelling law $\xi \sim a N_{\xi}^{v}$. Interactions between different blobs are screened out, and thus the chain is presumed to be ideal for distances large compared to $\xi$. The blob dimension (or the screening length) is given by [4]

$$
\xi \sim C^{-b}
$$

where $b=v /(v d-1)$. (We ignore unimportant prefactors.) Thus, the scaling exponent $b$ should take on values $3 / 4$ and $3 / 2$ in three- and two-dimensions, respectively [4].

In this letter we wish to present preliminary results of Monte-Carlo (MC) computer investigation of the behaviour of multiple chain systems $(d=2,3)$. The concentration dependence of the screening length will be examined and compared with the result of the scaling theory (2). The main result of our computation is : the first numerical confirmation of the equation (2).

2. Model and method. - The model and method of calculation were as described previously [5-7]. An $N$-mer was defined as a chain of hard spheres $(d=3)$ or hard circular disks $(d=2)$ of diameter $D=1$ indexed from 1 to $N$, which were connected by $N-1$ bonds of length $a=1$. The angles between neighbouring bonds were taken as $90^{\circ}$. The rotational angles were uniformly distributed for $d=3$ while for $d=2$ only the choice $\varphi= \pm \pi$ was possible.

The interactions between the non-bonded monomers were assumed to be given by the function :

$$
u_{\mathrm{ij}}= \begin{cases}0, & r_{\mathrm{ij}} \geqslant D \\ \infty, & r_{\mathrm{ij}}<D\end{cases}
$$

where $u_{\mathrm{ij}}$ is the interaction energy between a pair of monomers ( $\mathrm{i}$ and $\mathrm{j}$ ), with distance between them equal $r_{\mathrm{ij}}$. The potential $u_{\mathrm{ij}}$ represents the excluded volume interactions between different parts of polymer chains in a good solvent.

The $N$-mers were placed in a periodic box consistent with the fraction of the total possible volume occupied by monomers

$$
C=m N(D / L)^{d}
$$

where $m$ is the number of chains, and $L$ is the periodic box edge length. The volume fraction of the monomers was varied both by changing the size of the cell for a given number of $N$-mers and by keeping the size of the cell constant while changing the number of $N$-mers in the system. For each volume fraction (" concentration »), the results thus obtained were equivalent.

According to the prescription of reference [8] (see also de Gennes's book [4] (p. 83) and reference [7]), the value of $\xi$ (which corresponds to the spatial range of the concentration fluctuations) can be obtained from the equation

$$
C_{0}(\xi)=C
$$

where $C_{0}(r)$ is the intrachain contribution to the local volume fraction of monomers. We define $C_{0}(r)$ as an average number of monomers per unit volume $(d=3)$ or area $(d=2)$

$$
C_{0}(r) \sim\langle N(r)\rangle(D / r)^{d} .
$$

For each value of $C$, the entire sample of configurations of the system was generated by MC method in blocks of $10^{3}$ configurations. Final equilibrium averages $\langle N(r)\rangle$ and standard deviations were obtained by averaging over the block averages.

We shall not repeat the details of the calculational techniques. A detailed description of Monte-Carlo procedure used here has already been given in a number of interrelated publications [5-7]. This procedure was similar to that employed by Birshtein et al. [9]. 
3. Results. - Systems containing 40 unit chains $(N=40)$ were considered in this work. The volume fractions were ranged from 0.037 to $0.37(6 D \leqslant L \leqslant 60 D$ for the $3 d$ case and $21 D \leqslant L \leqslant 66 D$. for the $2 d$ case). The critical volume fractions $\left(C^{*}=N(D / R)^{d}\right)$ were estimated as $C^{*} \simeq 0.01(d=3)$ and $C^{*} \simeq 0.05(d=2)$ (for more detail see reference [6]). The values of $\xi$ were calculated from equations (5) and (6).

Graphical illustration of the behaviour of $\xi$ with respect to the variation in $C$ is given in figure 1 where $\ln \xi$ is plotted against $\ln C$ for $d=3$ and $d=2$. We note the linear dependences. When the polymer coils are isolated $\left(C<C^{*}\right)$, the values of $\xi$ are, of course, quite close to $R$. It can be seen from figure 1 that with increasing concentration, the values of $\xi$ decrease and approach the value of $D$. The decrease is more pronounced for the two-dimensional system.

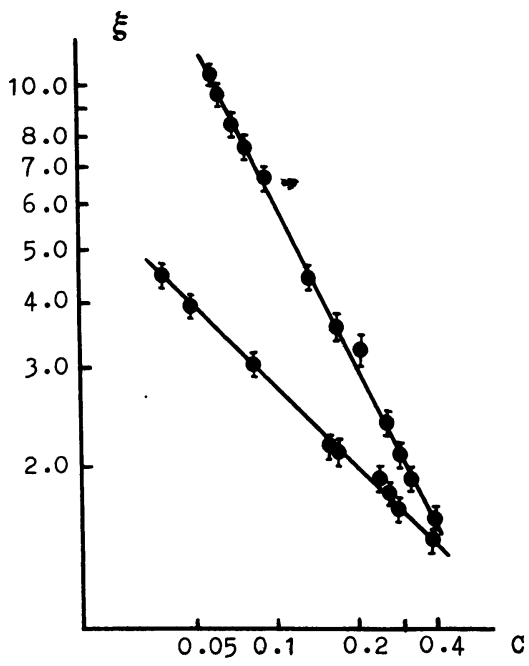

Fig. 1. - Least squares plots of $\ln \xi v s . \ln C$ for three-dimensional chains (lower curve) and two-dimensional chains (upper curve). In each case the curve represents a least squares fit of equation (7) to the data for those points corresponding to $C \geqslant 0.05$. The vertical bars represent the $90 \%$ confidence range.

The straight lines in the figure represent least squares best fits of equation (7) to the

$$
\xi=A(d) \cdot C^{-b} d
$$

data for $C \geqslant 0.05$. Analysis of variance for the three-dimensional screening length yielded the result

$$
b_{3}=0.65: \mathrm{CONF}_{90 \%}(0.57,0.73)
$$

where the numbers in brackets are the lower and upper $90 \%$ confidence limits, respectively, obtàined by multiplying the standard error $(\sigma=0.04)$ by 1.94 , the value of Student's $t$ for 6 degrees of freedom at the two-sided $90 \%$ confidence level. For $b_{2}$, the exponent for the twodimensional screening length, the standard error was found to be $\sigma=0.09$. The fitted slope can therefore be stated as $b_{2}=1.17 \pm 0.09$, or

$$
b_{2}=1.17: \mathrm{CONF}_{90 \%}(1.01,1.33) \text {. }
$$

These results can be compared with the predictions of the scaling theory (see equation (2)). The obtained exponents are qualitatively consistent with the expected exponents $b_{3}=3 / 4$ 
and $b_{2}=3 / 2$ [10]. The difference is not very large but, as we can see it, visible. (In both cases, the values for the exponent $b$ predicted by scaling theory $[4,8]$ are slightly outside the $90 \%$ confidence bar.)

One of the possible explanations for this divergence may be due to the fact that relatively short chains were considered. Of course, it would be of considerable interest to extend the calculations to longer chains. With the model used here, however, this is impractical, since at high densities the computer simulation of the long chains requires too much computer time. Another possible explanation $[11,6]$ is that for these short chains, the semidilute regime is not large enough to observe the exact scaling exponents. We hope to clarify these points in future numerical work.

Thus, the results of the computer simulation presented in this Letter provide support for the validity of the general scaling approach to the problem of the conformational statistics of flexible polymers in the semidilute regime of concentration.

In the publication being prepared the data will be presented in greater detail and subjected to further analysis.

Acknowledgments. - We would like to thank A. S. Pavlov and B. N. Klushnik for useful discussions and suggestions. We are also grateful to the referees (anonymous) of this paper for valuable criticism.

\section{References}

[1] De Gennes, P.-G., Phys. Lett. 38A (1972) 339.

[2] Des Cloizeaux, J., J. Physique 36 (1975) 281.

[3] The value of $y$ originally proposed by Flory $(v=0.6)$ has been recently recalculated using more sophisticated and exact arguments relying on renormalization theory with the result $v=0.588 \pm 0.001$ (Le Guillou, J. C., Zinn-Justin, J., Phys. Rev. Lett. 39 (1977) 95 ; BaKer, G. A., Nickel, B. G., Meiron, D. I., Phys. Rev. B 17 (1978) 1365.

[4] De Gennes, P.-G., Scaling Concepts in Polymer Physics (Cornell University Press) 1979.

[5] Khalatur, P. G., Vysokomol. soed. 22B (1980) 406.

[6] Khalatur, P. G., Pletneva, S. G., Vysokomol. soed. 24A (1982) 472.

[7] Khalatur, P. G., Pletneva, S. G., Vysokomol. soed. 24B (1982) (in press).

[8] Daoud, M., Cotton, J. P., Farnoux, B., Jannink, G., Sarma, G., Benoit, H., Duplessix, R., Picot, C., DE GenNes, P.-G., Macromolecules 8 (1975) 804.

[9] Birshtein, T. M., Skvortsov, A. M., Sariban, A. A., Vysokomol. soed. 19A (1977) 63.

[10] The obtained value : $b_{3}=0.65$ from $\xi_{3 d}$ is in better agreement with the scalling theory $\left(b_{3}=0.75\right)$ than with the mean field (Flory-Edwards) theory $(b=0.5)$.

[11] Curro, J. G., Macromolecules 12 (1979) 463. 